\title{
Heartbeat: Blood urea nitrogen to creatinine ratio predicts outcome in acute heart failure
}

In patients with acute heart failure (AHF), renal dysfunction is common and associated with a poor prognosis. Although both serum creatinine and blood urea nitrogen (BUN) are markers for renal dysfunction, BUN levels are also affected by reabsorption in the renal tubules, a process modulated by renin-angiotensin-aldosterone activity, sympathetic nervous activity and arginine-vasopressin activity. Thus, neurohormonal activity is reflected in the BUN/ creatinine ratio which effectively 'normalizes' BUN for the degree of renal dysfunction, as measured by the creatinine level. To investigate the prognostic value of the $\mathrm{BUN} /$ creatinine ratio in AHF, Matsue and colleagues $^{1}$ (see page 407) first defined age and sex specific normal ranges. Even in normal individuals, there is wide variation in the BUN/creatinine ratio, an increase with age, and higher levels in women compared to men. Then, in a cohort of about 2000 AHF patients, they showed that the BUN/creatinine ratio was elevated in about $25 \%$ and was a predictor of all cause death at 180 days (HR: 1.86, 95\% CI 1.29 to 2.66 ) and of death or cardiovascular or renal rehospitalisation at 60 days (HR: 1.37 , 95\% CI 1.03 to 1.82 ) after adjustment for other variables, including creatinine and BUN (figure 1).

In the accompanying editorial (see page 402), Núñez concludes: "Despite the encouraging results presented by Matsue et al, further studies are needed to confirm the normal/abnormal threshold for defining high/normal values of this ratio especially in older subjects, to define the clinical utility role of trajectories of BUN/ Creatinine over a single measurement, and to evaluate the prognostic utility in other subgroups of patients-underrepresented in this study - such as the elderly and patients with HF with preserved ejection fraction."

The hemodynamic changes with exercise in patients with pulmonary arterial hypertension provide important prognostic information and may be a target for therapeutic intervention, yet are

Correspondence to Professor Catherine M Otto, University of Washington, Division of Cardiology, Seattle,WA 98195, USA; cmotto@u.washington.edu incompletely understood. In an elegant and detailed study of 26 subjects with $\mathrm{PAH}$, Bellofiore and colleagues ${ }^{2}$ measured proximal and distal pulmonary artery stiffness, pulmonary impedance and other measures of ventricular-vascular coupling at rest and with exercise. Compared to normal subjects, PAH patients had increased pulmonary arterial stiffness at rest and a further increase in stiffness with exercise, despite no change in pulmonary vascular resistance (the more conventional measure). Increased pulmonary stiffness was associated with inefficient right ventricular to pulmonary artery coupling. PAH patients with more efficient hemodynamic coupling at rest, had greater pulmonary vascular reserve, allowing a higher cardiac output with exercise.

Claessen and La Gerche ${ }^{3}$ (see page 404) put these findings in perspective with a concise summary of normal and abnormal exercise hemodynamics (figure 2). They comment: 'Interestingly, it was found that coupling efficiency between the RV and the pulmonary circulation at rest was associated with maximum exercise tolerance whereas conventional metrics obtained by right heart catheterisation, such as pulmonary vascular resistance or cardiac output, were not. This underscores that in order to gain insights into the determinants of exercise tolerance we should not only assess the pulmonary vasculature, but also include its interaction with the right ventricle.'

The role of percutaneous intervention (PCI) versus coronary artery bypass grafting $(\mathrm{CABG})$ surgery in patients with multi-vessel coronary disease involving the proximal left anterior descending coronary artery remains controversial. In a pooled analysis of individual patient level data from the SYNTAX and BEST randomized trials, Cavalcante and colleagues ${ }^{4}$ (see page 428 ) found the primary composite outcome of all-cause death, myocardial infarction or stroke occurred in $16.3 \%$ of the PCI group and $11.5 \%$ of the CABG group at 5 years (HR $1.43 ; 95 \%$ CI 1.05 to $1.95 ; \mathrm{p}=0.026$ ), although all-cause death alone was not significantly different between the two groups. The authors suggest that 'Due to the sample size and randomised nature of the data, this study represents the most

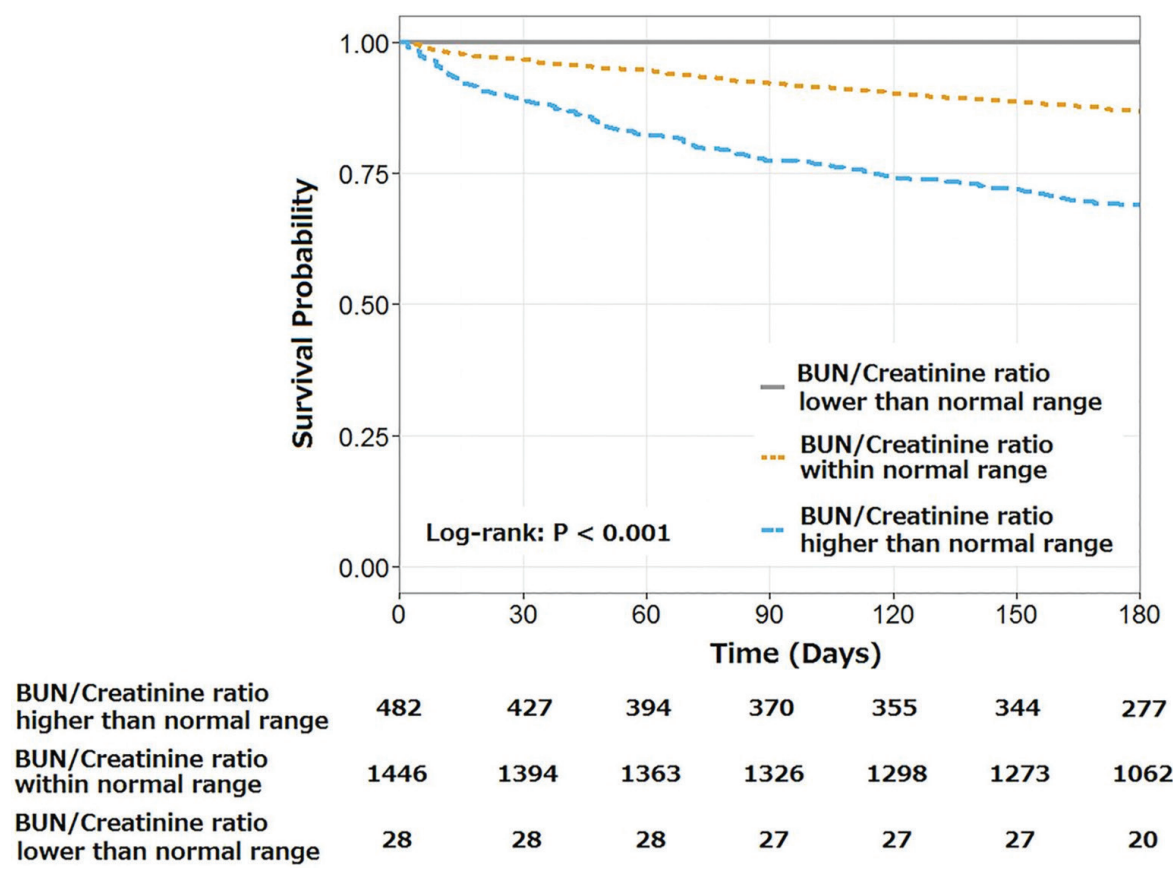

Figure 1 Kaplan-Meier survival curves for all-cause mortality within 180 days for the three different groups in patients with acute heart failure in the PROTECT study cohort. 


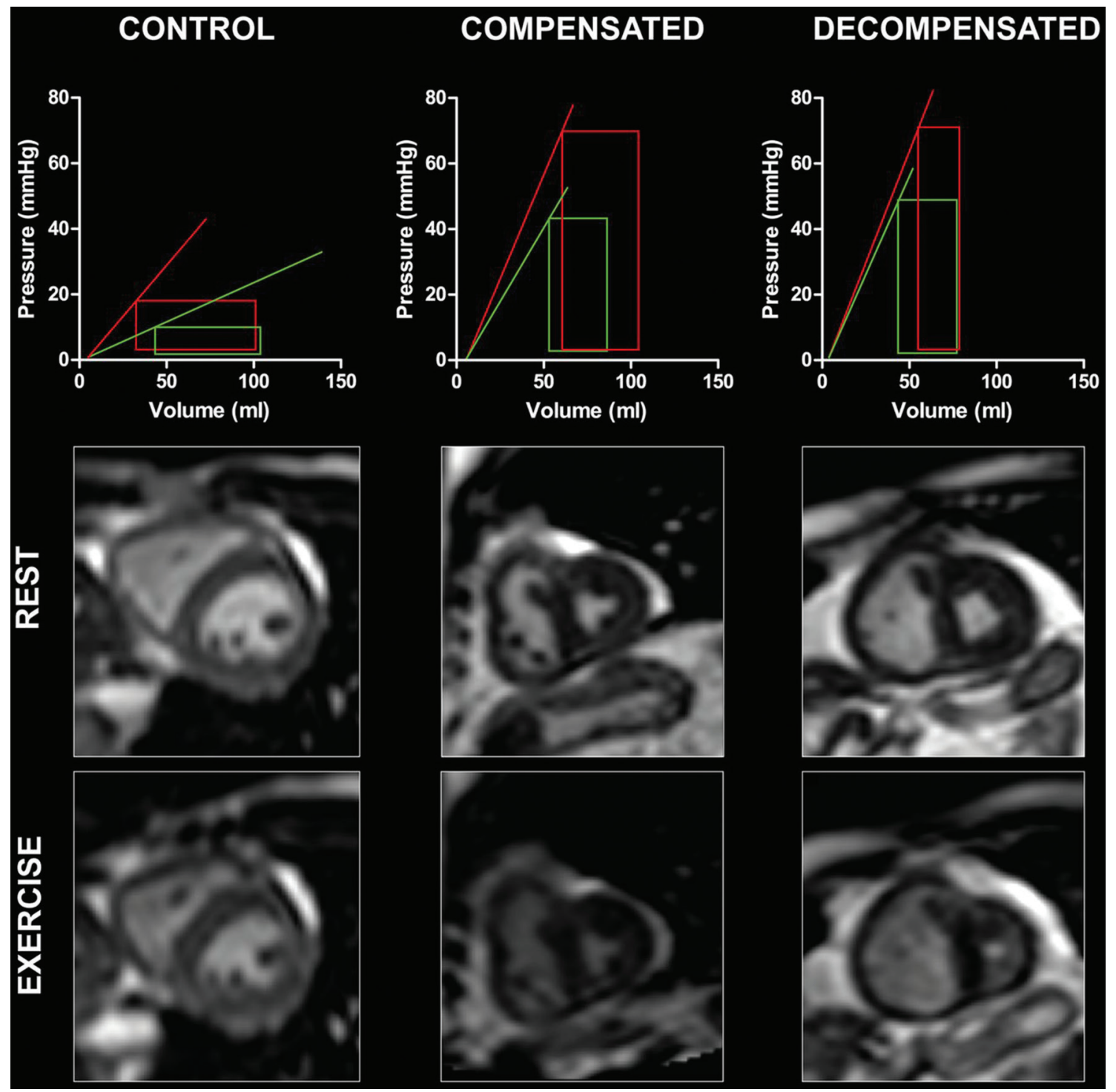

Figure 2 Illustration of exercise-induced changes in right ventricular-pulmonary arterial (RV-PA) coupling in a healthy subject, a patient with chronic thromboembolic pulmonary hypertension with 'compensated' disease and a patient with chronic thromboembolic pulmonary hypertension in the 'decompensated' stage of the disease. The upper panels show model right ventricle (RV) pressure-volume loops as previously computed by Houston and Tedford. Lines indicate end-systolic elastance (RV contractility) for each state (green=rest; red=exercise). Increasing slope of elastance denotes increasing contractility in this model. Note the control subject's ability to augment RV contractility with exercise, which decreases in the patient with compensated disease and is largely lost in the patient with pulmonary hypertension with decompensated disease. The lower panels depict midventricular slices at end systole obtained by cardiac MRI during exercise. RV end-systolic volume decreases normally in the healthy individual by the increase in RV contractility. In contrast, RV end-systolic volumes increase during exercise in the patients with chronic thromboembolic pulmonary hypertension, that is, RV-PA uncoupling. Note the dramatic increase in RV end-systolic volume in the patient with decompensated disease which results in D-shaping of the septum.

robust available data on this specific patient subset. Its results might impact practice guidelines to recommend CABG over PCI in patients with multi-vessel coronary disease and proximal LAD involvement.' Readers should send their comments about this recommendation to the authors using the online 'submit a response' option.

Also in this issue is an excellent review article entitled: 'Development of the atrial septum in relation to anatomy and interatrial communications' ${ }^{5}$ by Professor Anderson and colleagues (see page 456) which detailed evidence to correct some of the coomon clinical misconceptions about atrial anatomy (figure 3). The Education in Heart article $^{6}$ by D'Silva and Sharma (see page 463) discusses management of young competitive athletes with cardiovascular conditions which clinicians will find helpful in daily practice.

The Image Challenge article in this issue (see page 455) shows an excellent example of the use of coronary computed tomographic angiography for diagnosis in a young patient presenting with atypical chest pain.

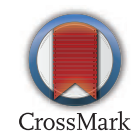

To cite Otto CM. Heart 2017;103:399-401.

Heart 2017:103:399-401.

doi: 10.1136/heartjnl-2017-311339 
A

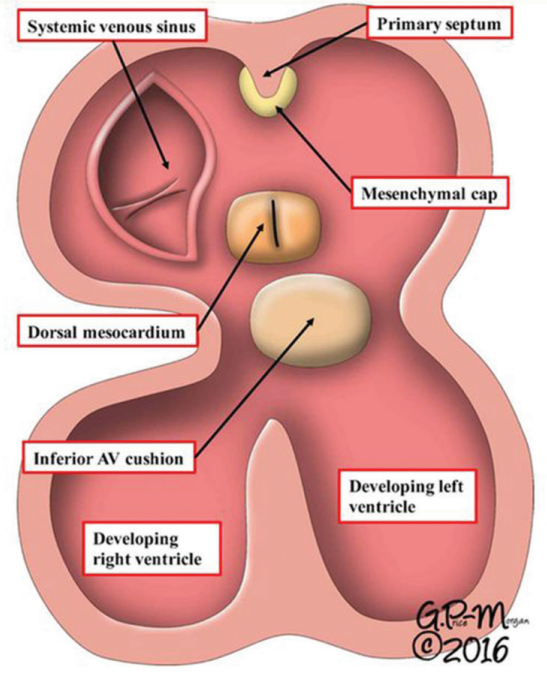

C

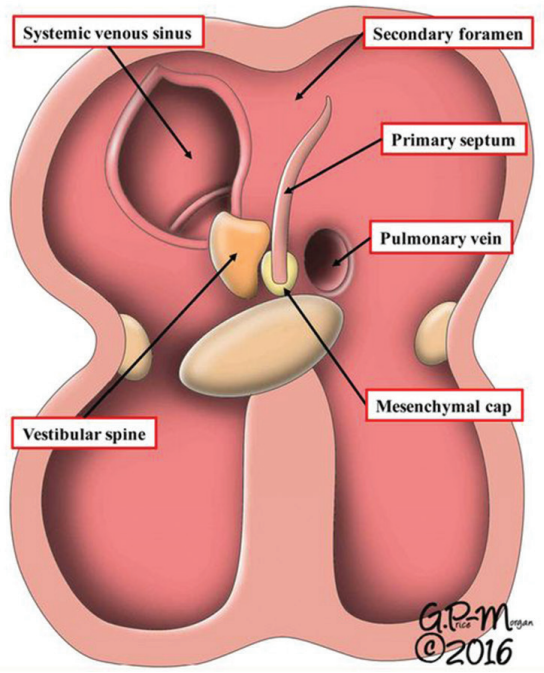

B

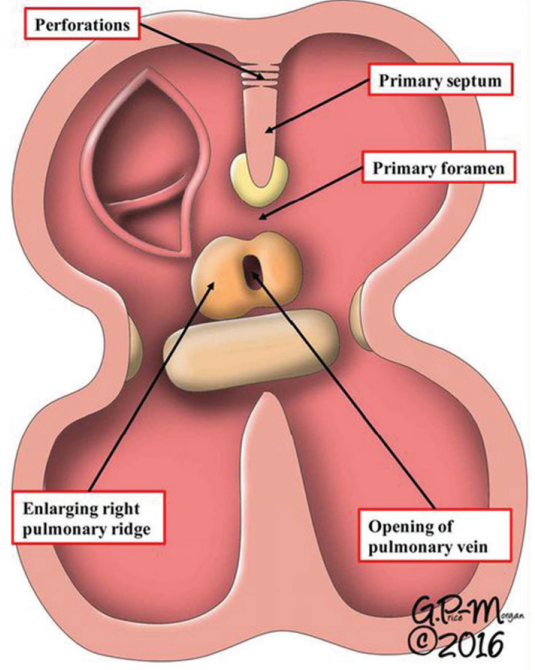

D

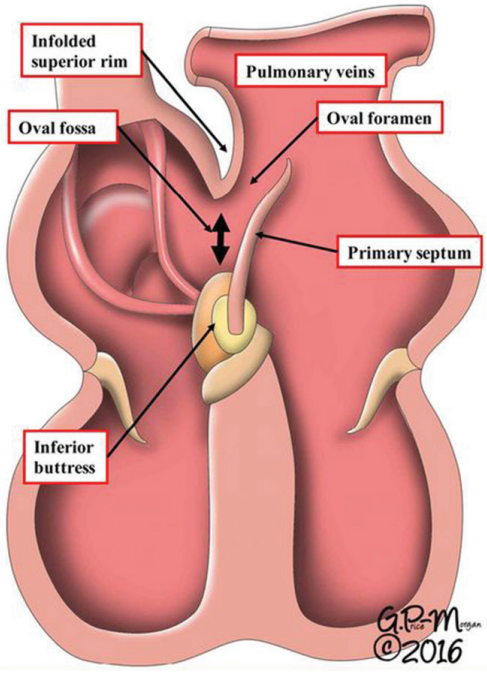

Figure 3 We have summarised the process of atrial septation in the human heart, showing the anatomical changes in the setting of a schematised dorsal half of the heart as its structure changes with the passage of time. In (A), we show the situation in the early embryonic stages, at the approximate 12th stage in the Carnegie categorisation, when there is effectively a common atrial cavity, with the systemic venous sinus already shifted so as to open to its right half. At this stage, a myocardial primary septum is seen growing from the atrial roof, covered by a mesenchymal cap. The dorsal mesocardium is a non-myocardial midline structure marking the remaining union between the atrial walls and the pharyngeal mesenchyme, with the reflections bulging into the atrial cavity as the left and the right pulmonary ridges, but with an imperforate pulmonary pit forming its floor. In (B), representing the time around the 16th Carnegie stage, we show that, as the primary septum grows towards the atrioventricular cushions, its increase in length progressively restricts the dimensions of the primary atrial foramen, with secondary perforations appearing close to its origin from the atrial roof. During this stage, the pulmonary vein has canalised within the pharyngeal mesenchyme, opening as a solitary structure into the left half of the atrial cavity through the pulmonary pit. In (C), which represents the end of the embryonic period, we show how the mesenchymal cap, the atrioventricular cushions and the vestibular spine, the latter being a derivative of the dorsal mesocardium, will fuse to close the primary atrial foramen. Concomitantly, the secondary perforations have coalesced to form the secondary atrial foramen. We illustrate the fetal stages in (D). The major change reflects the addition of pulmonary venous myocardium, with the initial solitary pulmonary venous opening migrating to the atrial roof. This change results in the development of a deep fold at the atrial roof, thus forming the superior rim of the oval fossa. The mesenchyme that closed the primary foramen will muscularise during the fetal stage, forming the inferior buttress of the oval fossa. The primary septum now forms the bottom, or flap valve, of the oval fossa, with the secondary foramen now recognisable as the oval foramen.

\section{REFERENCES}

1 Matsue $Y$, van der Meer P, Damman K, et al. Blood urea nitrogen-to-creatinine ratio in the general population and in patients with acute heart failure. Heart 2017; 103:407-13.

2 Bellofiore A, Dinges E, Naeije R, et al. Reduced haemodynamic coupling and exercise are associated with vascular stiffening in pulmonary arterial hypertension. Heart 2017;103:421-7.

3 Claessen G, La Gerche A. Stressing the right ventricular-pulmonary vascular unit: beyond pulmonary vascular resistance. Heart 2017;103:404-6.
4 Cavalcante $R$, Sotomi $Y$, Zeng $Y$, et al. Coronary bypass surgery versus stenting in multivessel disease involving the proximal left anterior descending coronary artery. Heart 2017;103:428-33.

5 Jensen B, Spicer DE, Sheppard MN, et al. Development of the atrial septum in relation to postnatal anatomy and interatrial communications. Heart 2017;103:456-62.

6 D'Silva A, Sharma S. Management of young competitive athletes with cardiovascular conditions. Heart 2017;103:463-73. 